\title{
Orites myrtoidea (Proteaceae): efecto de estratificación fría en germinación de semillas y ácido indol butírico en enraizamiento de estacas
}

\section{Orites myrtoidea (Proteaceae): effect of cold stratification in seed germination and indolebutyric acid on rooting of cuttings}

\section{Flavia Schiappacasse ${ }^{1, *}$, Carlos Peña ${ }^{1}$ \& Patricio Peñailillo ${ }^{2}$}

${ }^{1}$ Facultad de Ciencias Agrarias, Universidad de Talca, Talca, Chile.

${ }^{2}$ Instituto de Ciencias Biológicas, Universidad de Talca, Talca, Chile.

*Email: fschiap@utalca.cl

\section{RESUMEN}

Se estudió la propagación por semillas y por estacas de Orites myrtoidea ("Radal enano"), un arbusto con potencial ornamental de la familia Proteaceae, nativo de Chile y Argentina. A partir de material recolectado en otoño en la cordillera de la comuna de Pinto, Región de Ñuble, se evaluó el efecto en la germinación de semillas de 4 niveles de estratificación húmeda a $10^{\circ} \mathrm{C}(0,7,14$ y 21 días), más uno de ácido giberélico $(250 \mathrm{ppm})$ como control positivo, y el porcentaje y grado de enraizamiento de estacas con 5 niveles de inmersión rápida en solución de Ácido Indol Butírico (AIB) (0, 1.000, 2.000, 3.000 y 4.000 ppm) y un nivel de AIB en formulación en polvo (1.500 ppm). Los mayores porcentajes de germinación se obtuvieron con estratificación por 21 días y con inmersión en ácido giberélico, 80 y 92\%, respectivamente. En la propagación vegetativa el porcentaje de enraizamiento superó el $90 \%$ en todos los tratamientos, sin embargo, todas las estacas tratadas con AIB presentaron un enraizamiento moderado a abundante, superior al control, en el cual se obtuvo un enraizamiento escaso a moderado.

Palabras clave: AG, AIB, conservación ex situ, grado de enraizamiento, porcentaje de germinación, Proteaceae.

\section{ABSTRACT}

Propagation by seeds and cuttings was studied in Orites myrtoidea ("Dwarf Radal"), a shrub with ornamental potential that belongs to the Proteaceae family and it is native to Chile and Argentina. The plant material was collected in the autumn in the Andes mountain range from Pinto, Bío Bío Region. Four levels of moist stratification at $10^{\circ} \mathrm{C}(0,7,14$, and 21 days), and one level using Gibberellic acid ( $250 \mathrm{ppm})$ as a positive control, were tested to determine the effect on seed germination. The cutting's rooting percentages and grades were determined by testing 5 levels of quick dip in IBA liquid solution $(0 ; 1,000,2,000,3,000$ and 4,000 ppm) and one level using IBA in powder formulation (1500 ppm). The highest germination percentages were found with 21 days stratification and with immersion in Gibberelic acid, $80 \%$ and $92 \%$, respectively. In vegetative propagation the rooting percentage was over $90 \%$ in all treatments, however, all IBA-treated cuttings had a medium to abundant rooting, superior to the control, which had a scarce to moderate rooting.

Keywords: ex situ conservation, GA, germination percentage, IBA, Proteaceae, rooting grade. 


\section{INTRODUCCIÓN}

La familia Proteaceae posee un origen Gondwánico y actualmente se encuentra bien representada en Australia, África, Centro y Sudamérica, India, Nueva Zelandia y muchas islas del Pacífico y Oceanía (Rebelo 1995). Las especies de esta familia suelen crecer bien en suelos ácidos de baja fertilidad donde principalmente el fósforo y nitrógeno son muy escasos, debido a la presencia de raíces proteiformes o en glomérulos que les permiten obtener el fósforo retenido en el suelo (Rebelo 1995, Lambers et al. 2008). Dentro de la familia destacan las especies, variedades y cultivares de los géneros Leucadendron, Protea y Leucospermum, cuyo conocimiento biológico y agronómico es amplio. Poseen un follaje de tipo esclerófilo muy resistente a la deshidratación, y flores coloridas y llamativas, características útiles para su uso como follaje cortado, flor de corte y paisajismo (Matthews 2002, Rebelo 1995).

En Chile la familia está representada por 6 especies distribuidas en 4 géneros: Embothrium, Gevuina, Lomatia y Orites (Rodríguez et al. 2018). Su hábito va de árboles a arbustos y todas ellas presentan diverso potencial ornamental (Riedemann \& Aldunate 2003). El género Orites está presente en Chile y Argentina con la especie Orites myrtoidea (Poepp. \& Endl.) Benth. \& Hook. f. ex B.D. Jacks., un arbusto que posee un atractivo follaje, abundantes flores y decorativos frutos, lo cual asociado a su rusticidad la hacen recomendable para jardines, plazas y parques (Riedemann \& Aldunate 2003), aprovechando la tendencia que se viene observando desde la década de los 90 en el país de incorporar especies nativas al paisajismo (Teillier 2008).

Morfológicamente, O. myrtoidea es un arbusto perennifolio de porte bajo, muy ramificado y extendido que suele alcanzar los $2 \mathrm{~m}$ de altura por igual diámetro (Fig. 1). Las hojas de color verde claro brillante son de forma oblonga a elíptica, de textura coriácea y de 1,8-3,5 cm de largo por 0,6-1 cm de ancho (Hoffmann 1998, Hechenleitner et al. 2005, Riedemann \& Aldunate 2003). Las flores de color blanco en la antesis y amarillas cuando senescentes se agrupan en racimos densos y axilares de 1,5 $-2,5 \mathrm{~cm}$ de largo. Los frutos son folículos de color canela al madurar $y$, una vez secos, persisten en la planta (Fig. 2) (Riedemann \& Aldunate 2003; Hechenleitner et al. 2005; Rodríguez et al. 2007). La floración ocurre en primavera entre los meses de octubre y noviembre y la fructificación entre febrero y marzo (Hechenleitner et al., 2005). En su hábitat natural forma matorrales que crecen en las laderas rocosas de los Andes sobre suelos de origen volcánico desde los 760 a 2.100 m s.n.m. (Hechenleitner et al. 2005). Su área de distribución está fragmentada y existen registros de $\mathrm{O}$. myrtoidea desde la cordillera andina del Maule

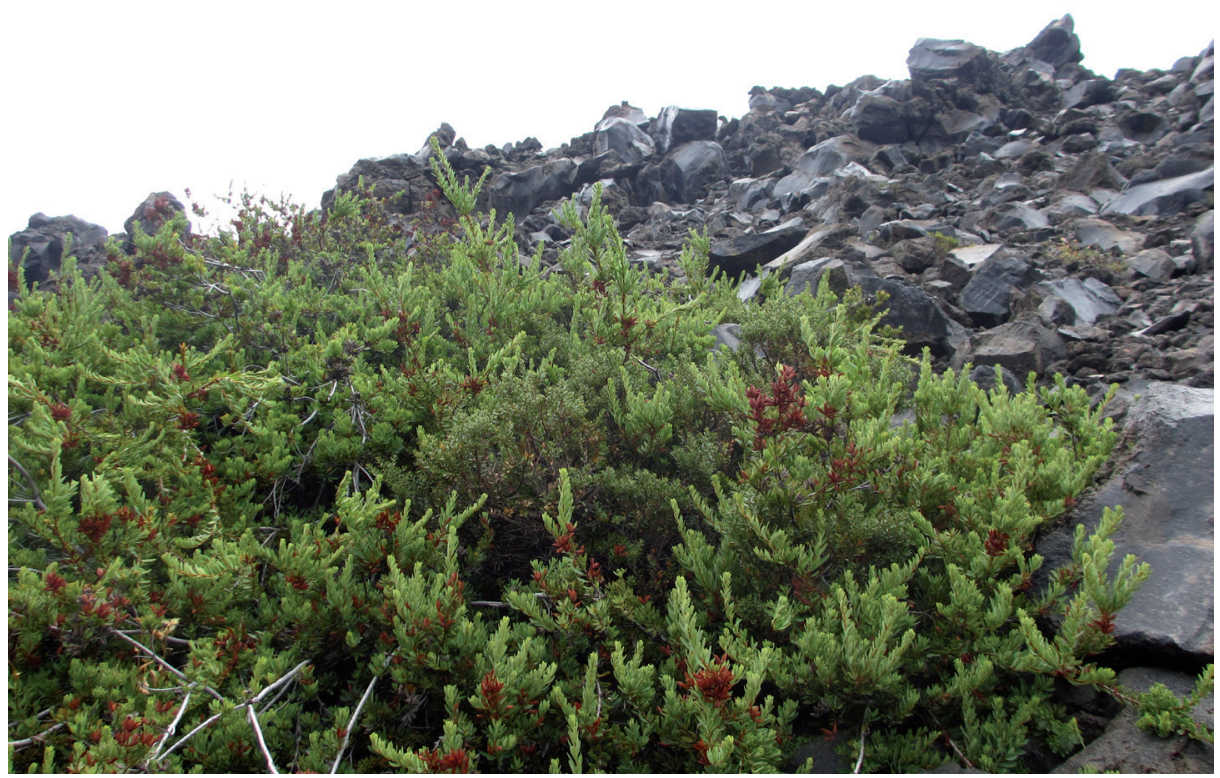

FiguRA 1. Ejemplar adulto de Orites myrtoidea creciendo sobre escoria volcánica en el sector andino de Shangri-La, Nevados de Chillán, Región de Ñuble. Destaca el hábito arbustivo, ramificado y extendido. / Adult plant specimen of Orites myrtoidea growing on volcanic slag in the Andean area of Shangri-La, Nevados de Chillan, Ñuble Region. Its spreading branches and extended shrubby growth habit are highlighted. 
hasta la de la Araucanía (Rodríguez et al. 2018). Según su estado de conservación se ha clasificado a la especie en la categoría de Casi Amenazada (NT) (Proceso Reglamento de Clasificación de Especies, № 9 DS 13/2013 MMA). No obstante, Echeverría \& Rodríguez (2014) señalan que en los últimos 10 años ha habido una reducción del área, extinción de poblaciones y algún grado de amenaza constante, por lo que proponen reclasificar esta especie como En Peligro, siguiendo los criterios de la UICN B1ab (i, iii, iv). Cabe hacer notar que sólo el $45 \%$ de la superficie que habita la especie se encuentra protegida en alguna entidad del Sistema Nacional de Áreas Silvestres Protegidas del Estado (SNASPE).

Ecológicamente, los arbustos colaboran en la estabilización del suelo, mantienen el balance hídrico, capturan el carbono atmosférico, sirven de alimento para animales y hongos, y actúan de plantas nodrizas favoreciendo a otras especies vegetales (Götmark et al. 2016). O. myrtoidea, como planta arbustiva de la Cordillera de los Andes, es importante al proveer estos beneficios. Al mismo tiempo, promocionar su cultivo como planta ornamental ayudaría a su conservación "ex situ", sobre todo si consideramos ésta como una estrategia actual para aquellas especies vegetales amenazadas, lo que implica mantener colecciones que estén disponibles para futuros programas de recuperación y conservación (Chacón et al. 2011, Saldías \& Velozo 2014).

O. myrtoidea es capaz de soportar condiciones adversas para el crecimiento, en su hábitat natural crece en condiciones de pleno sol y es capaz de resistir temperaturas bajo $0^{\circ} \mathrm{C}$. Estas características de rusticidad son ideales tanto para la promoción de su utilización en obras de paisajismo como en la restauración de paisajes y hábitats degradados, en especial en el área de distribución de la especie. Sin embargo, es necesario conocer aspectos de la propagación por semillas y vegetativa de la especie.

Se tienen antecedentes de la reproducción de la especie por semillas (Riedemann \& Aldunate 2003, Hechenleitner et al. 2005, Rodríguez et al. 2007), pero éstos son incompletos. Por otra parte, no existe información detallada de propagación por estacas, aunque se recomienda la aplicación de hormonas como enraizante (Riedemann \& Aldunate 2003). A la luz de los antecedentes expuestos el presente trabajo tiene como finalidad precisar los requerimientos en detalle de germinación de semillas y multiplicación por estacas para la especie 0 . myrtoidea.

\section{MATERIALES Y MÉTODOS}

El material vegetal se recolectó el día 1 de mayo de 2015 en un sector cordillerano de Chillán conocido como Shangri-La (36 $52^{\prime} 37^{\prime \prime} S$ y $71^{\circ} 27^{\prime} 47^{\prime \prime} W$ ), a 1.575 m s.n.m. Las semillas fueron almacenadas en seco hasta su utilización. Durante el ensayo se dispusieron en placas Petri, entre capas de papel filtro, humedecidas con solución al 0,2\% del fungicida Captan.

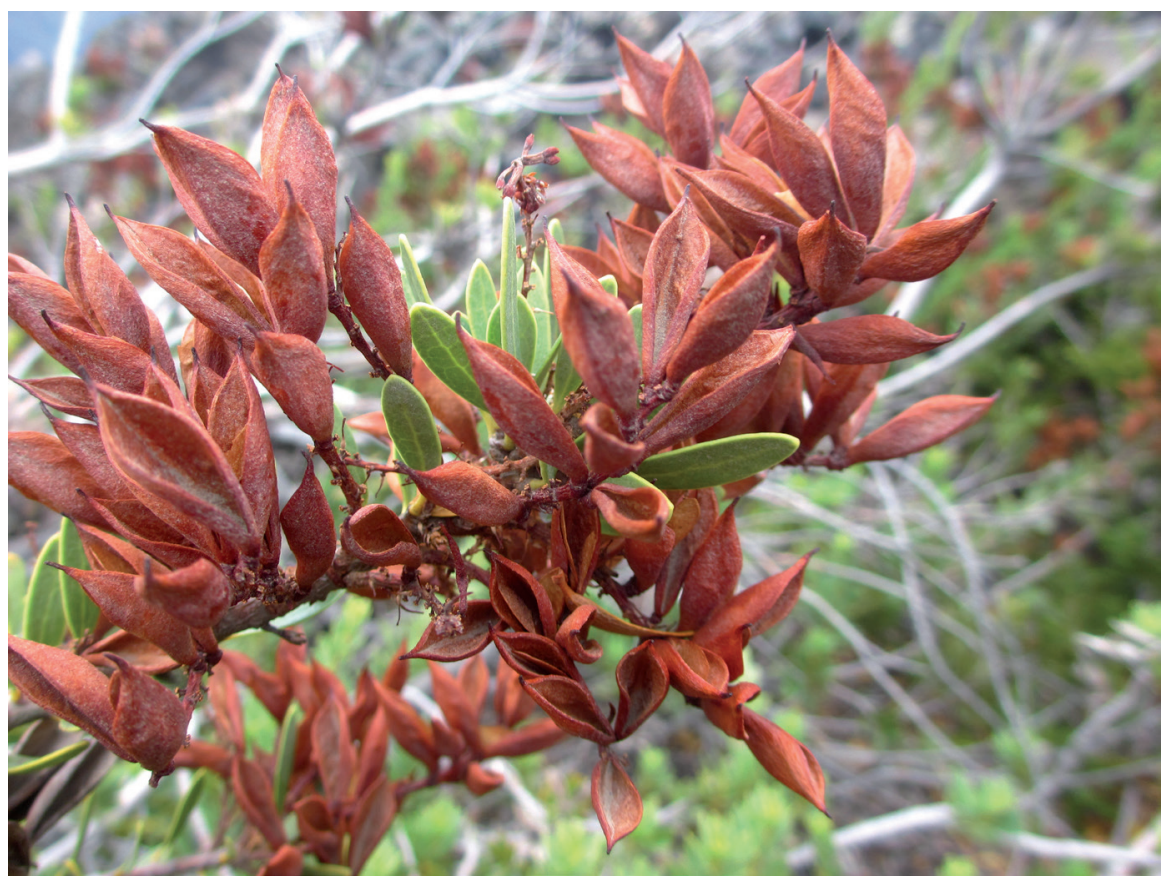

FIgURA 2. Frutos de Orites myrtoidea abiertos en otoño. / Open fruits of Orites myrtoidea in autumn. 
Los tratamientos aplicados fueron control (sin estratificación húmeda); remojo en solución de 250 ppm de ácido giberélico (control positivo) (Gibberellic acid en polvo, Merck) por 24 horas; y estratificación húmeda por 7, 14 y 21 días, los cuales fueron aplicados en forma secuencial, de modo que las semillas fueron puestas a germinar el 15 de octubre de 2015, en cámara de crecimiento a $20^{\circ} \mathrm{C}$ y bajo tubos fluorescentes blancos fríos. Se utilizaron 20 semillas por repetición, y un total de 5 repeticiones por tratamiento, bajo un diseño completamente al azar. Se evaluó la germinación diaria para obtener la germinación máxima acumulada, y para comparar los tratamientos que obtuvieron los mejores resultados, se calculó el Valor máximo de Czabator (1962) (máximo promedio diario de germinación), la Energía germinativa (porcentaje de germinación acumulado al día en que se produce el Valor máximo) y el $\mathrm{T}_{50}$ (tiempo para alcanzar el $50 \%$ de la germinación máxima) (Cabello et al. 2001-2002).

Para la propagación vegetativa se recolectaron ramillas de diferentes individuos, se dispusieron dentro de un envase aislante previa aspersión con agua para evitar su deshidratación. A su llegada a Talca fueron refrigeradas a $10^{\circ} \mathrm{C}$ hasta su utilización tres días después. Las estacas se dejaron de una longitud de $10 \mathrm{~cm}$, se les eliminaron el ápice y las hojas basales y se les aplicaron los distintos tratamientos. Se trasladaron de inmediato a un invernadero de estructura metálica con cobertura de polietileno, en la Estación Experimental Panguilemo de la Universidad de Talca, donde permanecieron en una cama de propagación con su base inserta en perlita, con calor basal (ajustado a $20^{\circ} \mathrm{C}$, pero sin funcionar entre 18:00 y 23:00) y bajo un sistema de nebulización intermitente controlada con Mist-A-Matic, hasta la evaluación del enraizamiento, aproximadamente tres meses después. Los tratamientos aplicados fueron: testigo (sin enraizante); enraizante a base de ácido indol butírico (AIB) (ANASAC, Chile) en polvo (1.500 ppm de AIB); inmersión por 5 segundos en solución de 1.000, 2.000, 3.000 y 4.000 ppm de AIB, preparadas a partir de solución alcohólica de 10.000 ppm de AIB (IBA Root Yates, de Distribuidora Yates Ltda.), diluyendo con acetona. Se aplicó un diseño completamente al azar, cada tratamiento con 3 repeticiones de 15 estacas cada una. Se evaluó el porcentaje de estacas enraizadas y su grado de enraizamiento (presencia y abundancia de raíces en base a una escala, en que $1=$ Nula presencia de raíces; 2 = Escasa; $3=$ Moderada; 4= Abundante) (Fig. 3).
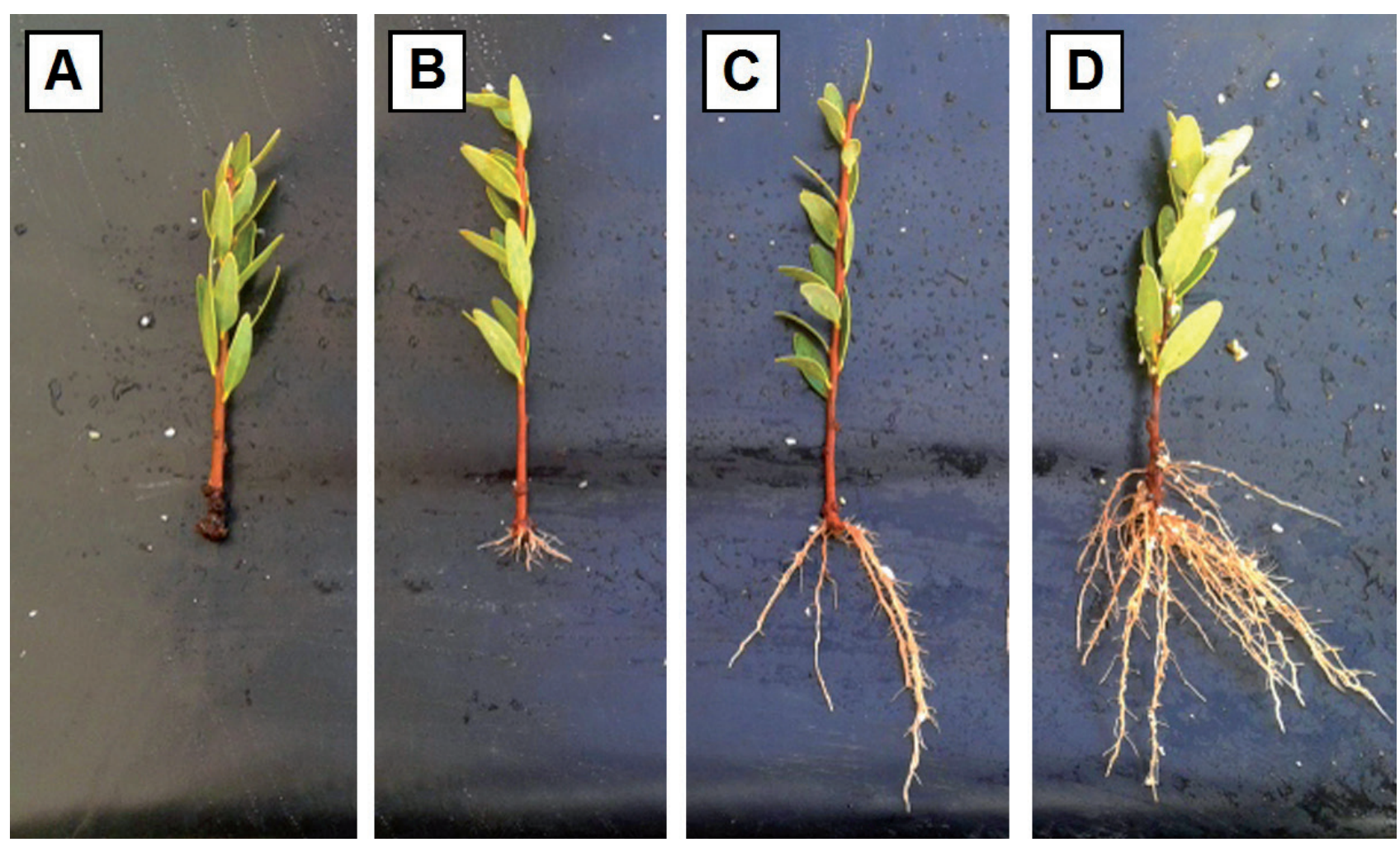

FıgURA 3. Grados de enraizamiento utilizados para la evaluación de estacas de Orites myrtoidea recolectadas en otoño. A) Grado 1: Nulo; B) Grado 2: Escaso; C) Grado 3: Moderado; D) Grado 4: Abundante. / Rooting grading for cuttings of Orites myrtoidea collected in the autumn. A) Grade 1: No rooting; B) Grade 2: Light rooting; C) Grade 3: Medium rooting; D) Grade 4: Heavy rooting 
Se realizó un análisis estadístico para los parámetros capacidad germinativa y valor máximo de Czabator, mediante un análisis de varianza y un test de comparación de medias (test HSD de Tukey) para determinar diferencias entre los tratamientos. En el caso del análisis del grado de enraizamiento se aplicó el test de Kruskal-Wallis. Se utilizó el programa estadístico Statgraphics Centurión XVI.II.

\section{RESULTADOS}

\section{GeRMINACIÓN DE SEMILLAS}

En todos los tratamientos se observó germinación. En el testigo sin estratificación y estratificación fría por 7 y 14 días, la capacidad germinativa fue baja, obteniéndose porcentajes que varían de 17 a 19\% (Tabla 1). En cambio, las semillas estratificadas por 21 días alcanzaron un $80 \%$ de germinación, es decir la capacidad germinativa fue alta. De los tratamientos de estratificación fría, la mayor energía germinativa evidenciada por el índice de Czabator fue en el tratamiento de 21 días de frío (31\%), lograda en 4 días. En el tratamiento de 14 días de frío se observó una baja energía germinativa (7\%), que se debe a que la germinación fue muy gradual, a la vez que muy baja.

El porcentaje de germinación con ácido giberélico fue de $92 \%$, no difiriendo significativamente del mejor tratamiento de estratificación en frío (21 días) (Tabla 1, Fig. 4). Las semillas estratificadas por 21 días iniciaron su germinación a los 2 días, y presentaron una curva de germinación adelantada con respecto a las del tratamiento de ácido giberélico, las cuales comenzaron a germinar desde los 10 días (Fig. 4), tardando las primeras 6 días para alcanzar el 50\% de la germinación máxima $\left(T_{50}\right)$, y en el caso de las semillas tratadas con ácido giberélico, 20 días. El valor máximo de Czabator alcanzado en ambos tratamientos fue de 7,75 al día 4, y de 2,93 al día 28, respectivamente.

\section{Propagación Vegetativa}

En la evaluación realizada después de 101 días, en todos los tratamientos el enraizamiento de los estacas superó el $90 \%$, incluyendo al testigo, no existiendo diferencias estadísticamente significativas entre ellos (Tabla 2). Sin embargo, se observa una tendencia a obtener mejores porcentajes de enraizamiento con hormona en forma líquida más que en polvo.

El grado de enraizamiento del testigo fue de escaso a moderado. Las estacas tratadas con hormona en formulación líquida variaron de moderado a abundante. También, se observó cualitativamente que el crecimiento de la especie es lento tanto en su hábitat natural como en las plántulas provenientes de las semillas y de estacas obtenidas en este estudio.

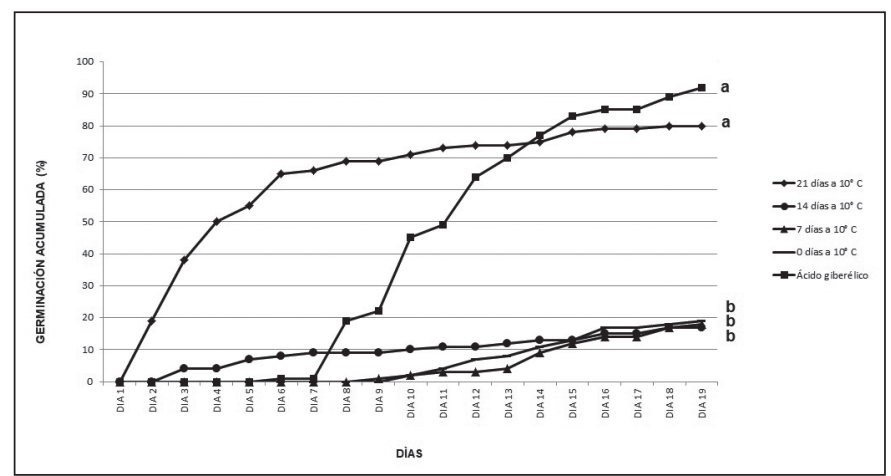

FiguRA 4. Germinación acumulada en semillas de Orites myrtoidea con distintos tratamientos pregerminativos. Valores acompañados de letras distintas difieren significativamente según test HSD de Tukey $(p<0,05)$. Cada valor es el promedio de 5 repeticiones de 20 semillas. / Cumulative germination in seeds of Orites myrtoidea with different pregermination treatments. Values with different letters are significantly different, according to HSD Tukey test $(p<0.05)$. Each value is a mean of 5 replicates of 20 seeds.

TABLA 1. Germinación de semillas de Orites myrtoidea bajo distintos periodos de estratificación a $10^{\circ} \mathrm{C}$ e inmersión en ácido giberélico (AG). / Germination of Orites myrtoidea seeds exposed to different stratification times at $10^{\circ} \mathrm{C}$ and immersion in gibberellic acid (GA).

\begin{tabular}{lccccc}
\hline Tratamiento & $\begin{array}{c}\text { Capacidad } \\
\text { Germinativa (\%) }\end{array}$ & $\begin{array}{c}\text { Valor Máximo } \\
\text { Czabator }\end{array}$ & $\begin{array}{c}\text { Energía } \\
\text { Germinativa (\%) }\end{array}$ & $\begin{array}{c}\text { Período de } \\
\text { energía (días) }\end{array}$ & $\mathrm{T}_{50}$ (días) \\
\hline Testigo & $19 \mathrm{~b}$ & $0,57 \mathrm{~b}$ & 17 & 30 & 27 \\
7 días de frío & $18 \mathrm{~b}$ & $0,50 \mathrm{~b}$ & 18 & 36 & 27 \\
14 días de frío & $17 \mathrm{~b}$ & $0,78 \mathrm{~b}$ & 7 & 9 & 12 \\
21 días de frío & $80 \mathrm{a}$ & $7,75 \mathrm{a}$ & 31 & 4 & 6 \\
AG 250 ppm/24 h & $92 \mathrm{a}$ & $2,93 \mathrm{~b}$ & 82 & 28 & 20 \\
\hline
\end{tabular}

Valores acompañados de letras distintas dentro de una columna difieren significativamente según test HSD de Tukey $(p<0,05)$. Cada valor es el promedio de 5 repeticiones de 20 semillas. / Values with different letters in a column are significantly different, according to HSD Tukey test $(p<0.05)$. Each value is a mean of 5 replicates of 20 seeds. 
TABLA 2. Enraizamiento de estacas de Orites myrtoidea con calor basal y nebulización intermitente, con o sin aplicación de ácido indol butírico (AIB), en invernadero frío al cabo de 101 días en otoño-invierno. / Rooting of Orites myrtoidea cuttings with basal heat and intermittent mist, with or without indole butyric acid (IBA) in an unheated greenhouse after 101 days in autumn-winter.

\begin{tabular}{lcc}
\hline Tratamiento & Porcentaje & Grado \\
\hline Testigo & 95,6 & $2,8 \mathrm{c}$ \\
Enraizante en polvo $^{1}$ & 91,1 & $3,3 \mathrm{~b}$ \\
$1.000 \mathrm{ppm}$ & 97,8 & $3,6 \mathrm{ab}$ \\
$2.000 \mathrm{ppm}$ & 100 & $3,9 \mathrm{a}$ \\
$3.000 \mathrm{ppm}$ & 95,6 & $3,6 \mathrm{ab}$ \\
$4.000 \mathrm{ppm}$ & 100 & $3,8 \mathrm{a}$ \\
Significancia & N.S. & \\
\hline
\end{tabular}

Valores en una columna seguidos por distinta letra difieren estadísticamente, según el test de Kruskal-Wallis ( $p<0.05$ ). Escala grado de enraizamiento: $1=$ Nulo; $2=$ Escaso; $3=$ Moderado; 4 = Abundante. ${ }^{1}$ Corresponde al enraizante de ANASAC, que contiene 1.500 ppm de AIB. / Values within a column followed by different letters are statistically different, according to Kruskal-Wallis test ( $p<0.05$ ). Rooting grading: 1 = No rooting; 2 = Light rooting; 3 : Medium rooting; 4 = Heavy rooting. ${ }^{1}$ Corresponds to rooting hormone ANASAC, that contains 1,500 ppm IBA.

\section{DISCUSIÓN}

\section{Germinación DE SEMILLAS}

En Chile, las especies arbóreas nativas de la familia Proteaceae, tales como Embothrium coccineum, Lomatia hirsuta y L. ferruginea que habitan los bosques templados del Sur de Chile presentan semillas dormantes (Donoso \& Escobar 1986). Nuestros resultados para O. myrtoidea, una especie arbustiva de los Andes de Chile Centro-Sur, muestran que las semillas requieren periodos de frío relativamente cortos (21 días) para romper la dormancia, similar a las semillas de las especies del género Lomatia antes mencionadas, las cuales requieren 15 a 45 días, en contraste con las de E. coccineum que necesitan más de 60 días (Donoso \& Escobar 1986).

El porcentaje de germinación de $80 \%$ obtenido con 21 días de estratificación a $10^{\circ} \mathrm{C}$ fue superior al $50 \%$ señalado para O. myrtoidea a $4^{\circ} \mathrm{C}$ por Rodríguez et al. (2007). Esta diferencia de la capacidad germinativa se pudo deber a la época de recolección de las semillas (otoño), las condiciones de conservación de las mismas, su edad al momento de inicio del periodo de frío, no descartando algún efecto de la temperatura de estratificación utilizada, entre otros. Por otra parte, es posible que con períodos de estratificación más largos, como por ejemplo 28 a 35 días, se obtenga una mayor germinación.

La germinación lograda mediante el uso de ácido giberélico de $92 \%$, no fue significativamente diferente a la lograda con estratificación por 21 días, lo que confirma lo señalado por Hechenleitner et al. (2005), en cuanto a que la aplicación de la hormona es un tratamiento recomendado para acelerar el proceso germinativo en O. myrtoidea. No obstante, si bien ambos tratamientos presentaron similar capacidad germinativa, al analizar la velocidad de germinación (Valor máximo de Czabator) y $\mathrm{T}_{50}$ (Tiempo para alcanzar el 50\% de la germinación máxima), el tratamiento de estratificación aparece como el más conveniente, al obtenerse un más rápido inicio de la germinación que con las semillas tratadas con ácido giberélico.

El comportamiento de germinación de las semillas de O. myrtoidea muestra que estaríamos ante semillas con dormancia fisiológica no profunda, según la clasificación de Baskin \& Baskin (2003), donde el ácido giberélico daría inicio a la actividad enzimática y luego de la imbibición de la semilla se activaría la movilización de los compuestos de reserva, que son proteínas, carbohidratos y lípidos (Hartmann et al. 2002), proceso que tarda unos días y explica la diferencia con el tratamiento de estratificación fría, en el cual las semillas se embebieron durante el proceso. En la naturaleza las semillas de O. myrtoidea se dispersarían durante el mes de otoño y al caer al suelo no germinarían de inmediato, sino que pasarían por una dormancia durante el período adverso de frío durante el invierno, germinando a fines de primavera, una vez que las condiciones sean favorables para la germinación y establecimiento de las plántulas.

\section{Propagación vegetativa}

En el presente estudio se logra un buen enraizamiento sin el uso de auxinas, pero el grado de enraizamiento del testigo sin auxinas fue inferior al resto de los tratamientos. Los mejores resultados en la formación de raíces adventicias se logran con 
concentraciones de AIB entre 1.000 a 4.000 ppm, similar a lo que sucede en especies de Proteaceae sudafricanas (Malan 1992) y la especie nativa de Chile G. avellana (Seemann et al. 2013). Existen diversos antecedentes que indican que una raíz más desarrollada resulta en un mejor desarrollo futuro de la planta (Hartmann et al. 2002).

El éxito del enraizamiento de estacas de O. myrtoidea obtenidas en otoño y puestas en cama caliente, se compara con aquel obtenido por Riedemann \& Aldunate (2003) al recolectar estacas en otra estación (primavera-verano) y en cama fría. Esto muestra que las estacas de esta especie se pueden recolectar en distintas épocas del año, siempre que el tejido caulinar esté fisiológicamente maduro, siendo la recomendación práctica para otras Proteáceas que el tallo se pueda doblar sin que se quiebre (Rebolledo \& Vico 2006).

\section{CONCLUSIONES}

La dormancia primaria que presentan las semillas de Orites myrtoidea, una especie amenazada del matorral arbustivo de la cordillera de los Andes del Centro-Sur, puede romperse para efectos de su propagación ex situ mediante estratificación húmeda por 21 días a $10^{\circ} \mathrm{C}$, o bien, mediante inmersión en ácido giberélico (AG). Vegetativamente, la especie puede propagarse por estacas con o sin la aplicación de hormona de enraizamiento (AIB), sin embargo es recomendable el uso de hormona para lograr un buen grado de enraizamiento.

Dado que la especie no presenta problemas de propagación ni por semillas ni estacas su conservación ex situ se ve favorecida, al igual que su uso en restauración de aquellos sitios donde crece en forma natural, donde su número ha sido mermado por acción antrópica.

La rusticidad de la especie, evidenciada por su capacidad para desarrollarse a plena luz y en suelos pobres en nutrientes, la convierten en una excelente candidata para jardines mediterráneos y cordilleranos de poco riego. Por otro lado, sus raíces en glomérulos, al aumentar el fósforo disponible en la zona de la rizosfera, favorecerían la nutrición de especies cercanas y/o especies que se establezcan con posterioridad, siendo una especie pionera y nodriza, ideal para proyectos de restauración en su área de distribución.

\section{AGRADECIMIENTOS}

A la Sra. Ana Sandoval, del Banco base de semillas del Centro experimental de INIA Intihuasi de Vicuña, por sus sugerencias y apoyo bibliográfico. A la Estación experimental Panguilemo de la Universidad de Talca por facilitar sus instalaciones. A los revisores anónimos por su contribución a mejorar el manuscrito.

\section{REFERENCIAS}

Baskin, J.M., Baskin, C.C. 2003. Classification, biogeography, and phylogenetic relationships of seed dormancy. In: Smith, R.D., Dickie, J.B., Linington, S.H., Pritchard, H.W., Probert, R.J. (eds.), Seed conservation: turning science into practice, Chapter 28, pp. 521-544. The Royal Botanic Gardens, Kew, London, United Kingdom.

Cabello, A., Sandoval, A., Carú, M. 2001-2002. Efecto de los tratamientos pregerminativos y de las temperaturas de cultivo sobre la germinación de semillas de Talguenea quinquenervia (Talguén). Ciencias Forestales 16(1-2): 11-18.

Chacón, P., Lagos-Witte, S., Mora, A., Moraes, M. 2011. Manual para la implementación de la "Estrategia Global para la Conservación de las Especies Vegetales" (EGCEV) en América Latina: El aporte de la Red Latinoamericana de Botánica al objetivo 1, meta 2. Red Latinoamericana de Botánica. Primera Edición, Santiago, Chile. 60 pp.

Czbator, F.J. 1962. Germination value: an index combining speed and completeness of pine seed germination. Forest Science 8(4): 386-396.

Donoso, C., Escobar, B. 1986. Germinación de las Proteáceas Arbóreas Chilenas. Bosque (Valdivia) 7 (2): 85-94.

Echeverría, C., Rodríguez, R. 2014. Caracterización de Eucryphia glutinosa, Citronella mucronata, Prumnopitys andina y Orites myrtoidea según los criterios de la UICN. Informe Final Fondo de Investigación del Bosque Nativo. Concepción, Chile. 72 pp.

Götmark, F., Götmark, E., Jensen, A.M. 2016. Why a shrub? A hypothesis for the adaptive value of a common growth form. Frontiers in Plant Science 7(1095): 1-14.

Hartmann, H.T., Kester, D.E. Davies, F.T., Geneve, R. 2002. Plant Propagation principles and practices. $8^{\text {th }}$ Edition. Prentice Hall, New Jersey, U.S.A. 915 pp.

Hechenleitner, P., Gardner, M.F., Thomas, P.I., Echeverría, C., Escobar, B., Brownless, P., Martínez, C. 2005. Plantas Amenazadas del Centro-Sur de Chile. Distribución, Conservación y Propagación. Primera edición. Universidad Austral de Chile y Real Jardín Botánico de Edimburgo, Chile. 187 pp.

Hoffmann, A. 1998. Flora silvestre de Chile, zona araucana. Cuarta Edición Revisada. Santiago, Chile. 258 pp.

Lambers, H., Raven, J.A., Shaver, G.R., Smith, S.E. 2008. Plant nutrient-acquisition strategies change with soil age. Trends Ecology and Evolution 23(2): 95-102.

Malan, D.G. 1992: Propagation of Proteaceae. Acta Horticulturae 316: 27-34.

Matthews, L.J. 2002. The Protea Book. A guide to cultivated Proteaceae. Canterbury University Press, Christchurch, 
New Zealand. 184 pp.

Rebelo, T. 1995. Proteas. A field guide to the Proteas of Southern Africa. Fernwood Press. South Africa. 224 pp.

Rebolledo, P., Vico, V. 2006. Propagación. En: Schiappacasse, F., Rebolledo, P., Herrera, R. (eds.), Cultivo comercial de Proteáceas en Chile, pp. 30-35. Editorial Universidad de Talca, Talca, Chile.

Riedemann, P., Aldunate, G. 2003. Flora nativa de valor ornamental, Chile Zona Sur, Identificación y Propagación. Primera Edición. Editorial Andrés Bello. Santiago de Chile.

Rodríguez, G., Rodríguez, R., Barrales, H.L. 2007. Plantas Ornamentales Chilenas. Segunda Edición. Editora Gráfica Lamas. Concepción, Chile. 236 pp.
Rodríguez, R., Marticorena, C., Alarcón, D., Baeza, C., Cavieres, L., Finot, V.L., Fuentes, N., Kiessling, A., Mihoc, M., Pauchard, A., Ruiz, E., Sánchez, P., Marticorena, A. 2018. Catálogo de las plantas vasculares de Chile. Gayana Botánica 75(1): 1-430.

Saldías, G., Velozo, J. 2014. Estudio de la propagación de Myrcianthes coquimbensis (Barnéoud) Landrum et Grifo por semillas y esquejes. Gayana Botánica 71(1): 17-23.

Seemann, P., Kramm, R., Awad, G., Ojeda, I., Vásquez, M.H. 2013. Rooting responses of some Chilean Proteaceae with ornamental potential. Acta Horticulturae 990: 429-435.

Teillier, S. 2008. Plantas de Chile en parques y jardines del mundo. Chloris Chilensis 11(2). Online. URL: http:// www.chlorischile.cl. Accedido: Enero 15, 2018.

Received: 19.12 .2018

Accepted: 21.04.2019 